\title{
Melissopalynological and physicochemical analysis of honey samples from Prayagraj District, Uttar Pradesh
}

\author{
VIBHASA SHUKLA ${ }^{1 *}$ and AJAY KUMAR ${ }^{2}$ \\ ${ }^{1}$ Department of Botany, University of Delhi, Delhi - 110007, India; e-mail: vib.shukla19@gmail.com \\ ${ }^{2}$ Botanical Survey of India, CRC, Prayagraj - 211002, India; e-mail: kajay4281@gmail.com
}

Received 27 September 2019; accepted for publication 19 March 2020

\begin{abstract}
In melissopalynological and physicochemical analyses of 14 honey samples collected from different rural and urban localities of Prayagraj District, Uttar Pradesh, 43 pollen types were identified and categorized as predominant (above 45\%), secondary (16-45\%), important minor (3-15\%) and minor (below 3\%). Five of the 14 samples were classified as unifloral due to the presence of predominant pollen types (above 45\%), and the other 9 as multifloral. Brassica campestris and Coriandrum sativum were the predominant pollen types in unifloral honey; 14 pollen types were documented in the secondary pollen types (16-45\%) in multifloral honey. The analyzed physicochemical parameters included $\mathrm{pH}$ (mean $3.40 \pm 0.15$ to $4.74 \pm 0.4$ ), electrical conductivity (mean $0.13 \pm 0.03$ to $1.39 \pm 0.17 \mathrm{mS} / \mathrm{cm}$ ), total dissolved solids $(120 \pm 1.23$ to $1260 \pm 1 \mathrm{ppm})$, moisture content $(12.17 \pm 1.39$ to $24.78 \pm 1.54 \mathrm{mg} / 100 \mathrm{~g})$ and ash content $(0.15 \pm .04$ to $1.68 \pm .27 \mathrm{mg} / 100 \mathrm{~g})$. The color of the honey ranged from water-white to dark amber. Among the minerals, magnesium was found to be most abundant $(9 \pm 0$ to $11.8 \pm 0 \mathrm{mg} / \mathrm{kg})$, followed by iron $(2.0 \pm 0$ to $4.8 \pm 0 \mathrm{mg} / \mathrm{kg}) \mathrm{and}$ zinc $(0.39 \pm 0$ to $0.63 \pm 0 \mathrm{mg} / \mathrm{kg})$. The heavy metals cadmium, lead, copper and arsenic were below the limit of detection $(<0.01 \mathrm{mg} / \mathrm{kg})$ in all honey samples except in samples H6 (Cd $0.01 \mathrm{mg} / \mathrm{kg}, \mathrm{Pd} 0.27 \mathrm{mg} / \mathrm{kg}), \mathrm{H} 11(\mathrm{Cd} 0.02 \mathrm{mg} / \mathrm{kg}$, $\mathrm{Pd} 0.05 \mathrm{mg} / \mathrm{kg}), \mathrm{H} 13(\mathrm{Pd} 0.02 \mathrm{mg} / \mathrm{kg}$ ) and H14 (Cd $0.01 \mathrm{mg} / \mathrm{kg}, \mathrm{Pd} 0.04 \mathrm{mg} / \mathrm{kg})$. The physicochemical parameters varied significantly $(\mathrm{p}<0.05)$ between samples. Findings of the present study indicate that the honey is of good quality and should be used in projects for commercialization of regional honey.
\end{abstract}

KEYWORDS: melissopalynology, multifloral honey, physicochemical analysis, unifloral honey

\section{INTRODUCTION}

Melissopalynology is a branch of palynology that deals with pollen analysis of honey. Pollen analysis provides relevant information about the plants preferred by honey bees as sources of pollen and nectar, and also aids in determining the geographical and botanical origin of the honey (Cotte et al., 2004; Ponnuchamy et al., 2014). This information is useful in developing apiaries and commercial honey production. Honey is a natural carbohydrate-rich product produced by honey bees from the nectar of plants. Honey possesses valuable nutritive, healing and prophylactic properties (Pereira

Corresponding author et al., 1995). The botanical and geographical origin of honey is related to the floral sources, soil, environmental conditions, and mode of extraction and processing (El-Metwally, 2015).

Melissopalynological and physicochemical analyses of honey from India have been published by Khatija and Ramanujan (1993), Nanda et al. (2003), Cherian et al. (2011), Ramnath and Venkataramegowda (2012), Gairola et al. (2013) and Shobham et al. (2017), but a literature survey indicates that for honey samples from Prayagraj District there are only a few pollen analyses (Sahney and Rahi, 2015; Sahney et al., 2018). To help fill the gap, we determined melissopalynological and 
physicochemical parameters $(\mathrm{pH}$, electrical conductivity, total dissolved solids, moisture content, ash content, color, mineral content) of honey produced by Apis dorsata bees from different rural and urban localities of Prayagraj District, Uttar Pradesh.

\section{MATERIALS AND METHODS}

\section{PALYNOLOGICAL ANALYSIS}

Prayagraj District lies in northwestern Uttar Pradesh, India (Fig. 1). Fourteen squeezed winter honey samples of Apis dorsata were collected from hives in rural localities (Kotwa, H1, H9; Phaphamau, H10; Phoolpur, H5, H12; Handia, H11) and urban localities (Company garden, H4, H8; Govindpur, H2, H13; Lukarganj, H7; Naini, H6; Teliyarganj, H3, H14) in Prayagraj District in 2017-2018 by professionals under our supervision, then stored in airtight plastic bottles and labeled. During field surveys of the plants growing around the bee hive sites, floral materials were collected for preparation of reference slides, which were made according to the method of Wodehouse (1935). Pollen grain analyses followed the method recommended by the International Commission of Bee Botany (Louveaux et al., 1978). Ten grams of honey were weighed and dissolved in $20 \mathrm{ml}$ distilled water, the solution was centrifuged, the supernatant was decanted and the sediments were placed on a slide. After drying, the sediments were mounted with glycerin jelly, covered with a cover slip and examined under a light microscope. Four pollen slides were made from each honey sample, and 300 or more pollen grains were counted from each slide at random, for a total of 1200 or more pollen grains per sample. Pollen recovered from the honey samples was identified against reference pollen slides. Pollen counts were made at random, covering the maximum mounted area to avoid repetition. Once identified and counted, the pollen grains were assigned to frequency classes: predominant pollen type (above 45\%), secondary pollen type (16-45\%), important minor pollen type (3-15\%) and minor pollen type $(<3 \%)$ (Louveaux et al., 1978). Honey samples containing more than $45 \%$ of a single predominant pollen type were categorized as unifloral, and samples with no predominant pollen type as multifloral. Based on frequency of occurrence in total honey samples, pollen types were classified as very frequent ( $>50 \%)$, frequent $(20-50 \%)$, infrequent (10-20\%) and rare $(<10 \%)$ (Feller-Demalsy et al., 1987). Absolute pollen counts (APCs) of honey samples (number of pollen grains per $10 \mathrm{~g}$ honey) were calculated using a haemocytometer (Suryanarayana et al., 1981). Following Louveaux et al. (1978), honey samples were assigned to various groups: Gp I (APC <20000), Gp II (APC 20000-100000), Gp III (APC 100000-500000), Gp IV (APC 500000-1000000) and Gp V (APC >1000000). These pollen count groups correspond to extremely poor, poor rich, very rich and extremely rich, respectively, as in Feller-Demalsy et al. (1989).

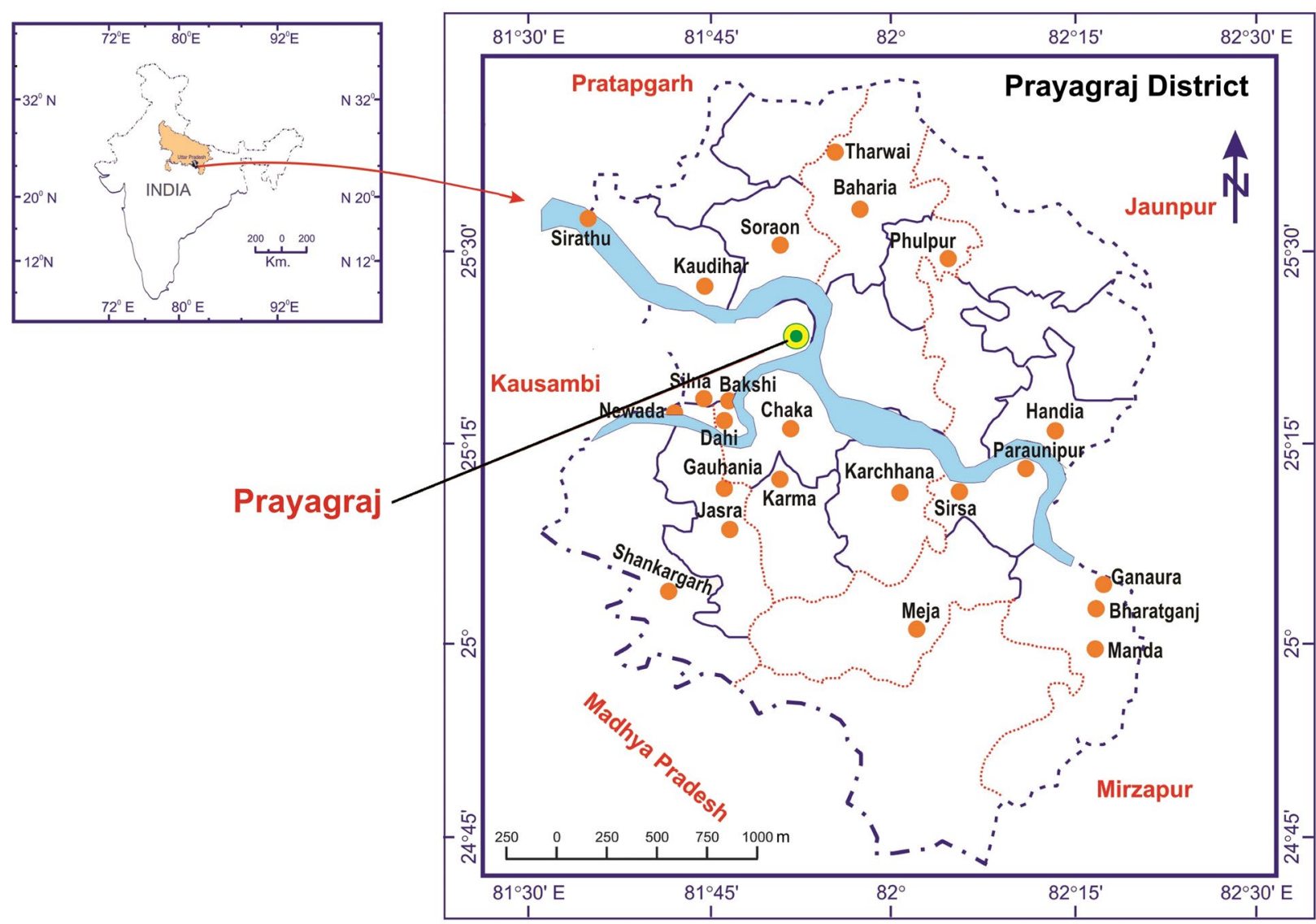

Fig. 1. Map showing the study area in Prayagraj District, Uttar Pradesh 


\section{PHYSICOCHEMICAL ANALYSIS}

Physicochemical analyses of honey samples followed international recommendations (Bogdanov et al., 1997; AOAC 1999), using the following methods:

- Color was determined with a UV-VIS spectrophotometer (RIGOL - Ultra 3400, single-beam-based) by reading absorbance in aqueous solutions at $635 \mathrm{~nm}$ (10 $\mathrm{g}$ honey in $20 \mathrm{ml}$ water). Table 2 shows honey colors and their mm Pfund values, obtained using the following algorithm (Bianchi, 1990): $\mathrm{mm}$ Pfund = $-38.7+371.39 \times$ absorbance.

- $\mathrm{pH}$ was determined with a digital $\mathrm{pH}$ meter (Labronics LT-49) from solutions prepared by dissolving $10 \mathrm{~g}$ honey in $75 \mathrm{ml} \mathrm{CO}$-free distilled water.

- Electrical conductivity (EC) and total dissolved solids (TDS) were determined with a digital EC/TDS meter (Labronics LT-51) in solutions made by dissolving $20 \mathrm{~g}$ honey in $100 \mathrm{ml} \mathrm{CO}$ free-distilled water.

- Ash content was determined according to AOAC (1999) using a muffle furnace (Thermotech Tic-4000). Three grams (g) of honey sample were weighed in a dry silica crucible. The weighed samples were gently heated in a muffle furnace until the sample became black and dry. Then the samples were ignited at $550^{\circ} \mathrm{C}$ for three to five hours. To ensure completion of ashing, it was reheated again in the furnace for half an hour (ash becomes white or grayish white). The ash was cooled in desiccators and weighed.

$$
\text { Ash content }=\frac{\mathrm{w}_{1}-\mathrm{w}_{2}}{\mathrm{w}_{0}} \times 100
$$

where,

$\mathrm{w}_{0}=$ weight of honey taken

$\mathrm{w}_{1}=$ weight of dish + ash

$\mathrm{w}_{2}=$ weight of dish

- For moisture content, $3 \mathrm{~g}$ of a sample was placed in a pre-weighed flat-bottom dish and kept overnight in a hot-air oven at $100-110^{\circ} \mathrm{C}$ and weighed. The loss in weight was taken as the measure of moisture content, calculated by the following formula:

$$
\text { Moisture }(\%)=\frac{\mathrm{w}_{1}-\mathrm{w}_{2}}{\mathrm{w}_{1}} \times 100
$$

where,

$\mathrm{w}_{1}=$ weight of fresh honey sample

$\mathrm{w}_{2}=$ weight of dry honey sample

\section{DETERMINATION OF MINERALS AND HEAVY METALS}

Minerals ( $\mathrm{Mg}, \mathrm{Fe}, \mathrm{Zn})$ and heavy metals $(\mathrm{Cd}, \mathrm{Pb}$, $\mathrm{Cu}, \mathrm{As}$ ) were determined in a certified laboratory (University of Allahabad, Prayagraj) using an atomic absorption spectrometer (Perkin Elmer Analyst 700).

\section{STATISTICAL ANALYSIS}

All physicochemical analyses were done in triplicate, and the data presented as means and standard deviations. Statistical analyses employed SPSS ver. 16. Significance was calculated by one-way analysis of variance (ANOVA) followed by Duncan's multiple range test $(\mathrm{p}<0.05)$

\section{RESULTS AND DISCUSSION}

We identified 43 pollen types belonging to 26 families in the 14 honey samples collected from rural and urban localities in Prayagraj District, Uttar Pradesh, during the 2017-2018 winter season (Tab. 1, Fig. 2). Table 2 presents the obtained palynological data for the honey samples.

Thirty-five of the 43 pollen types were found to be from entomophilous plant taxa, two pollen types were found to be from amphiphilous taxa, and six pollen types were found to be from anemophilous taxa (Tab. 1). The 35 entomophilous taxa are $A$. conyzoides, $A$. indica, A. leptopus, B. campestris, B. ceiba, Bougainvillea sp., C. cajan, Calliandra sp., Callistemon sp., C. fistula, Chrysanthemum sp., C. arietinum, Citrus sp., C. sativum, C. indicum, D. sisso, D. stramonium, Dianthus sp., $H$. rosa-sinensis, $H$. auriculata, J. simplex, Lathyrus sp., L. esculentum, $M$. oleifera, O. sanctum, P. hysterophorus, $P$. zeylanica, $P$. guajava, $R$. sativus, $S$. arvensis, Tagetes sp., T. stans, T. grandifolia, Verbena sp. and Vernonia sp. The anemophilous taxa are A. indica, Chenopodium sp., H. integrifolia, Solanum sp., Cyperaceae and Poaceae; presumably they were incidentally transported by wind or were inadvertently transported by bees to the hives. The two amphiphilous taxa we identified are $M$. alba and E. citriodora.

The main nectariferous families we documented were Acanthaceae (3 pollen types), Apiaceae (1), Asteraceae (6), Bignoniaceae (1), Bombacaceae (1), Brassicaceae (2), Caryophyllaceae (1), Combretaceae (1), Fabaceae (6), Lamiaceae (1), Malvaceae (1), Meliaceae (1), Moraceae (1), Moringaceae (1), Myrtaceae (3), Nyctaginaceae (1), Plumbaginaceae (1), Polygonaceae (1), Rutaceae (1), Solanaceae (3) and Verbenaceae (1). We documented five nectarless plant families: Chenopodiaceae, Cyperaceae, Euphorbiaceae, Poaceae and Ulmaceae (Tab. 1; Figs 3, 4).

Five of the 14 honey samples were classed as unifloral, due to the presence of predominant $B$. campestris (samples $\mathrm{H} 1, \mathrm{H} 5, \mathrm{H} 8, \mathrm{H} 10$ ) and $C$. sativum (H9). Nine honey samples were classed as multifloral (H2, H4, H6, H7, H8, H11, H13, H14). Fourteen secondary pollen types were recorded in multifloral honey samples: A. conyzoides, A. leptopus, A. indica, B. ceiba, B. campestris, C. cajan, C. fistula, Callistemon sp., Chenopodium sp., C. sativum, E. citriodora, $M$. oleifera, $P$. hysterophorus and $P$. guajava. 
Table 1. Vernacular name, habit, flowering period, mode of pollination and sources of plant species. AM - Amphiphilous taxa; AN - Anemophilous taxa; EN - Entomophilous taxa; N - Nectariferous; P - Polliniferous

\begin{tabular}{|c|c|c|c|c|c|}
\hline Plant species & Vernacular/Common name & Habit & $\begin{array}{c}\begin{array}{c}\text { Flowering } \\
\text { period }\end{array} \\
\end{array}$ & $\begin{array}{c}\text { Mode of } \\
\text { pollination }\end{array}$ & Sources \\
\hline \multicolumn{6}{|l|}{ Acanthaceae } \\
\hline Hygrophila auriculata & Marsh barbel & Herb & Sep-Feb & EN & NP \\
\hline Justicia simplex & Common justicia & Herb & Aug-Mar & EN & NP \\
\hline Thunbergia grandifolia & Neel lata/Bengal trumpet vine & Liana & Sep-Jan & EN & NP \\
\hline \multicolumn{5}{|l|}{ Apiaceae } & NP \\
\hline \multicolumn{6}{|l|}{ Asteraceae } \\
\hline Ageratum conyzoides & Tropical whiteweed & Herb & Jan-Dec & EN & NP \\
\hline Chrysanthemum sp. & Guldaudi & Herb & Oct-Jan & EN & NP \\
\hline Parthenium hysterophorus & Gajar ghas/Carrot grass & Herb & Jan-Dec & EN & NP \\
\hline Sonchus arvensis & Milk thistle & Herb & Sep-Mar & EN & NP \\
\hline Tagetes sp. & Marigold & Herb & Dec-Feb & EN & NP \\
\hline Vernonia sp. & Sadodi & Herb & Mar-Apr & EN & NP \\
\hline \multicolumn{6}{|l|}{ Bignoniaceae } \\
\hline Tecoma stans & Yellow trumpetbush & Tree & Jan-Dec & EN & NP \\
\hline \multicolumn{6}{|l|}{ Bombacaceae } \\
\hline Bombax ceiba & Semul & Tree & Feb-Mar & EN & NP \\
\hline \multicolumn{6}{|l|}{ Brassicaceae } \\
\hline Brassica campestris & Mustard & Herb & Nov-Apr & EN & NP \\
\hline Raphnus sativus & Radish & Herb & Nov-Apr & EN & NP \\
\hline \multicolumn{6}{|l|}{ Caryophyllaceae } \\
\hline \multicolumn{6}{|l|}{ Chenopodiaceae } \\
\hline \multicolumn{6}{|l|}{ Combretaceae } \\
\hline Combretum indicum & Madhu malati & Liana & Oct-Mar & EN & NP \\
\hline Cyperaceae & Cyperaceae & Herb & Jan-Dec & AN & $\mathrm{P}$ \\
\hline \multicolumn{6}{|l|}{ Euphorbiaceae } \\
\hline Acalypha indica & Kuppi & Herb & Sep-May & $\mathrm{AN}$ & $\mathrm{P}$ \\
\hline \multicolumn{6}{|l|}{ Fabaceae } \\
\hline Cajanus cajan & Tuvar/Pigeon pea & Herb & Oct-Mar & EN & NP \\
\hline Calliandra sp. & Red powder puff & Shrub & Jan-Dec & EN & NP \\
\hline Cassia fistula & Amaltas & Tree & Jan-Oct & EN & NP \\
\hline Cicer arietinum & Chickpea & Herb & Oct-Mar & EN & NP \\
\hline Dalbergia sisso & Shisham & Tree & Oct-Mar & EN & NP \\
\hline Lathyrus sp. & Pulse & Herb & Jan-Apr & EN & NP \\
\hline \multicolumn{6}{|l|}{ Lamiaceae } \\
\hline Ocimum sanctum & Tulsi & Herb & Mar-Apr & EN & $\mathrm{N}$ \\
\hline \multicolumn{6}{|l|}{ Malvaceae } \\
\hline Hibiscus rosa-sinensis & Gurhal & Shrub & Jan-Dec & EN & NP \\
\hline \multicolumn{6}{|l|}{ Meliaceae } \\
\hline Azadirachta indica & Neem & Tree & Jan-Mar & EN & $\mathrm{N}$ \\
\hline Moraceae & & & & & \\
\hline Morus alba & Shahtoot/White mulberry & Tree & Feb-Jun & $\mathrm{AM}$ & NP \\
\hline Moringaceae & & & & & \\
\hline Moringa oleifera & Sajina & Tree & Feb-Apr & EN & $\mathrm{N}$ \\
\hline Myrtaceae & & & & & \\
\hline Eucalyptus citriodora & Eucalyptus & Tree & Dec-Apr & $\mathrm{AM}$ & NP \\
\hline Psidium guajava & Guava & Tree & Aug-Sep & EN & NP \\
\hline Callistemon sp. & Bottle brush & Shrub & Jan-Dec & EN & NP \\
\hline Nyctaginaceae & & & & & \\
\hline Bougainvillea sp. & Booganbel & Shrub & Jan-Dec & EN & NP \\
\hline Plumbaginaceae & & & & & \\
\hline Plumbago zeylanica & Chitrak & Herb & Jan-Apr & EN & NP \\
\hline Poaceae & Grass family & Herb & Jan-Dec & AN & $\mathrm{P}$ \\
\hline Polygonaceae & & & & & \\
\hline Antigonon leptopus & Coral vine & Liana & Aug-Mar & $\mathrm{AM}$ & NP \\
\hline Rutaceae & & & & & \\
\hline Citrus sp. & Lemon & Shrub & Feb-May & EN & $\mathrm{N}$ \\
\hline Solanaceae & & & & & \\
\hline Datura stramonium & Dhatura & Herb & Nov-May & $\mathrm{EN}$ & NP \\
\hline Lycopersicon esculentum & Tomato & Herb & Jan-Apr & EN & NP \\
\hline Solanum sp. & Solanum & Herb & Jun-Dec & $\mathrm{EN}$ & NP \\
\hline Ulmaceae & & & & & \\
\hline Holoptelea integrifolia & Chilbil & Tree & Jan-Mar & AN & $\mathrm{P}$ \\
\hline Verbenaceae & & & & & \\
\hline Verbena sp. & Barbena & Herb & Oct-Apr & EN & NP \\
\hline
\end{tabular}




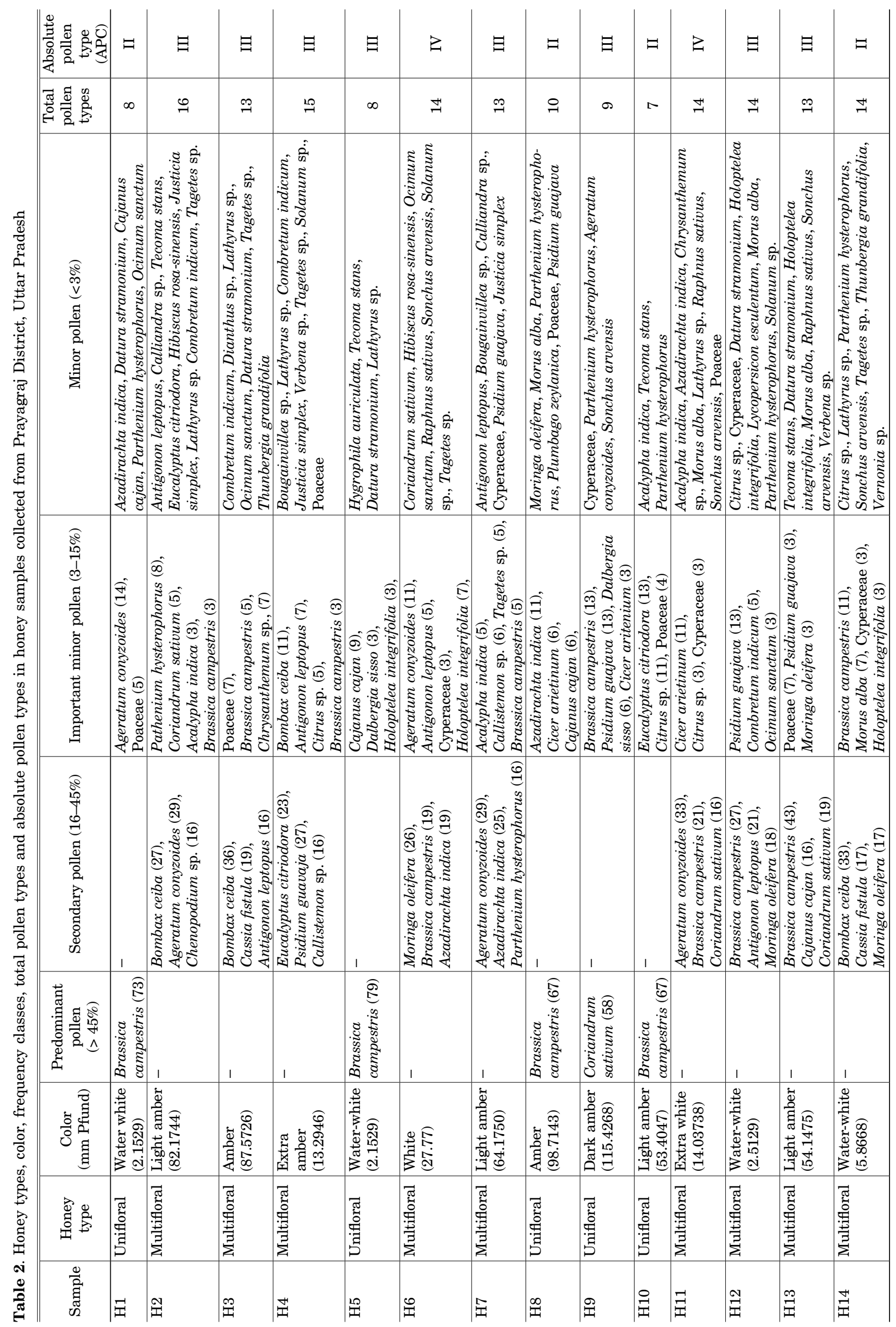


$\mathrm{H1}$

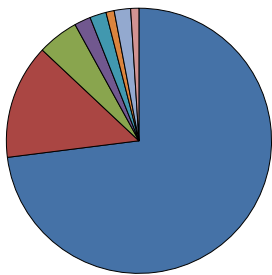

Brassica campestris

Poaceae

Datura stramonium

Parthenium hysterophorus

Ageratum conyzoides

Azadirachta indica

Cajanus cajan

Ocimum santum

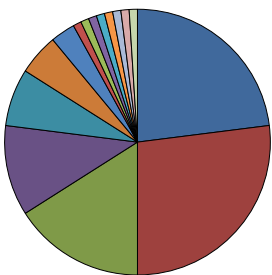

Eucalyptus citriodora $\quad$ Psidium guavaja

Callistemon sp.

Antigonon leptopus

Brassica campestris

Lathyrus sp.

Justicia simplex

Tagetes sp.

Poaceae

Bougainvillea sp.

Combretum indicum

Verbena sp.

Solanum sp.
$\mathrm{H} 2$

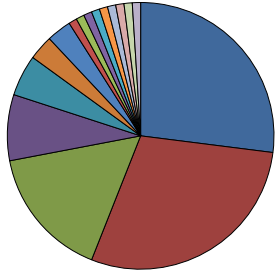

Bombax ceiba ageratum conyzoides

Chenopodium sp. 1 Pathenium hysterophoru

Coriandrum sativum Acalypha indica

Brassica campestris Antigonon leptopus

Calliandra sp. - Tecoma stans

Eucalyptus citriodora Hibiscus rosa-sinensis

Justicia simplex Lathyrus sp.

Combretum indicum Tagetes sp.

$\mathrm{H} 5$

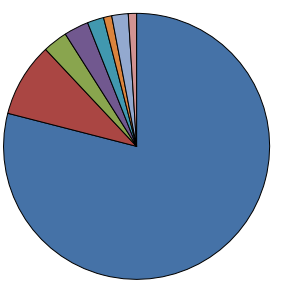

Brassica campestris Cajanus cajan

Dalbergia sisso $\quad$ Holoptelea integrifolia

Tecoma stans $\quad$ Hygrophila auriculata

Datura stramonium $\square$ Lathyrus $\mathrm{sp}$.

\section{$\mathrm{H} 7$}

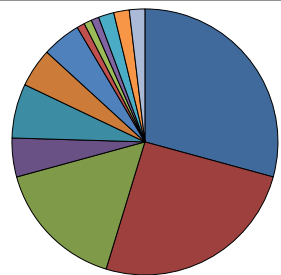

Ageratum conyzoides $\square$ Azadirechta indica Parthenium hysterophorus Acalypha indica

- Callistemon sp. Tagetes sp.

Brassica campestris

Bougainvillea sp.

Antigonon leptopus

Cyperaceae

Calliandra sp.

Psidium guajava

- Justicia simplex

$\mathrm{H} 10$

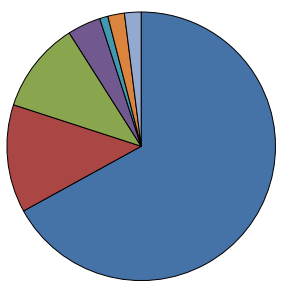

Brassica campestris Eucalyptus citriodora

Citrus sp.

Acalypha indica

Parthenium hysterophorus

\section{Poaceae}

Tecoma stans
$\mathrm{H} 8$

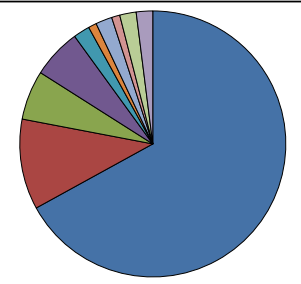

Brassica campestris Morus alba

Cicer arietinum Plumbago zeylanica

Moringa oleifera $\square$ Psidium guajava

Parthenium hysterophorus

Poaceae

Azadirachta indica

Cajanus cajan

$\mathrm{H} 11$

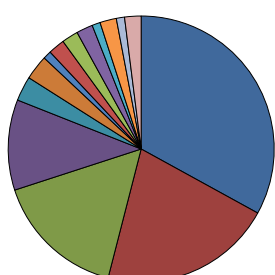

Ageratum conyzoides Brassica campestris

Coriandrum sativum Cicer arietinum

Citrus sp. $\quad$ Cyperaceae

Acalypha indica Azadirachta indica

Chrysanthemum sp. Morus alba

Lathyrus sp. $\quad$ Raphnus sativus

Sonchus arvensis Poaceae
H3

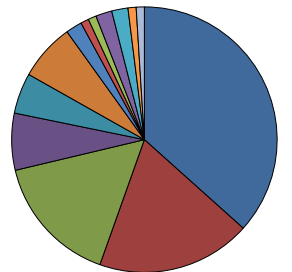

Poaceae

Bombax ceiba

Chrysanthemum sp.

Antigonon leptopus Chrysanthe
Brassica campestris Dianthus sp.

Combretum indicum Ocimum sanctum

Lathyrus sp.

Tagetes sp.

Datura stramonium

Thunbergia grandifolia

- Cassia fistula

\section{$\mathrm{H} 6$}

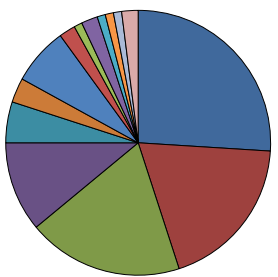

Moringa oleifera Brassica campestris

Azadirachta indica Ageratum conyzoides

Antigonon leptopus Cyperaceae

Holoptelea integrifolia Coriandrum sativum

Hibiscus rosa-sinensis Ocimum sanctum

Raphnus sativus Sonchus arvensis

Solanum sp. $\quad$ Tagetes sp.

\section{$\mathrm{H} 9$}

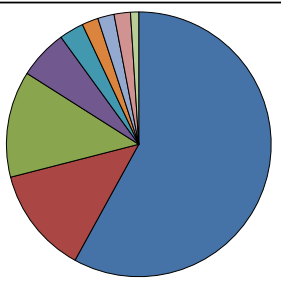

Coriandrum sativum Dalbergia sisso

Psidium guajava @yperaceae

- Cicer arietinum Ageratum conyzoides

Parthenium hysterophorus

Sonchus arvensis

Brassica campestris

\section{$\mathrm{H} 12$}

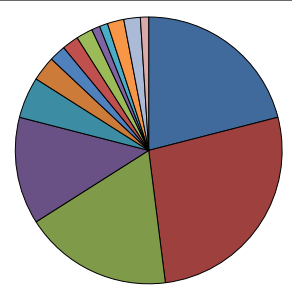

Antigonon leptopus Brassica campestris

Moringa oleifera Psidium guajava

Combretum indicum Ocimum sanctum

Citrus sp. $\quad$ Cyperaceae

Datura stramonium Holoptelea integrifolia

Lycopersicon esculentum Morus alba

Parthenium hysterophorus Solanum sp.

\section{$\mathrm{H} 13$}

Brassica campestris

Coriandrum sativum

- Psidium guajava

- Tecoma stans

Holoptelea integrifolia

Raphnus sativus Moringa oleifera

Verbena sp. Datura stromonium

- Cajanus cajan Morus alba

- Poaceae Sonchus arvensis
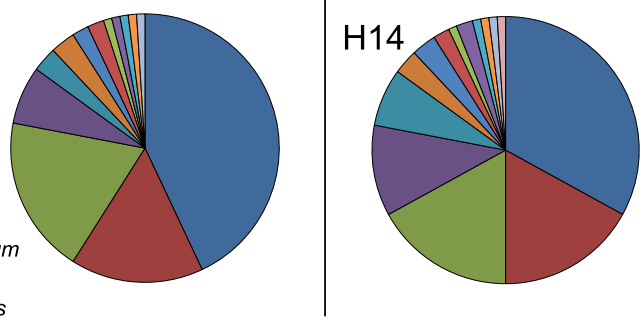

\begin{tabular}{ll}
\hline Bombax ceiba & Cyperaceae \\
Moringa oleifera & Citrus sp. \\
Morus alba & Parthenium \\
Holoptelea integrifolia & hysterophorus \\
Lathyrus sp. & Tagetes sp. \\
Sonchus arvensis & Vernonia sp. \\
Thunbergia grandifolia & \\
Cassia fistula & \\
Brassica campestris
\end{tabular}

Fig. 2. Pollen spectra of each honey sample collected from different localities in Prayagraj District, Uttar Pradesh 


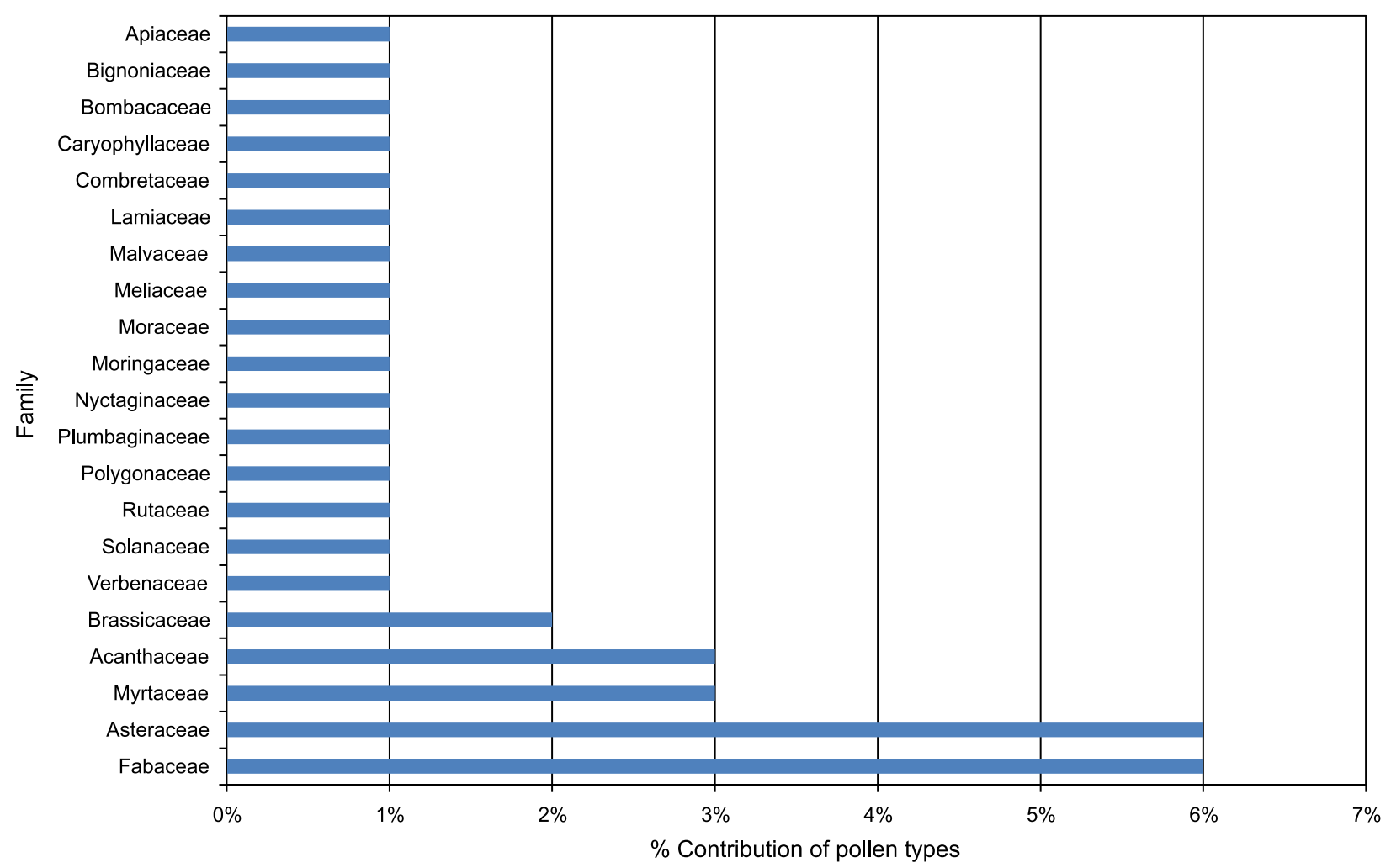

Fig. 3. Contribution per family of nectariferous pollen types

Twenty-four pollen types were recorded as important minor types of the pollen content: A. indica, A. conyzoides, A. leptopus, A. indica, B. campestris, B. ceiba, C. cajan, Callistemon sp., C. fistula, Chenopodium sp., Chrysanthemum sp., C. arietinum, Citrus sp., C. indicum, C. sativum, Cyperaceae, D. sisso, E. citriodora, $H$. integrifolia, M. oleifera, M. alba, O. sanctum, $P$. hysterophorus, $P$. guajava and Poaceae.

Thirty-four pollen types were recorded as minor components: A. indica, A. leptopus, A. indica, Bougainvillea sp., C. cajan, Calliandra sp., T. stans, Chrysanthemum sp., Citrus sp., C. indicum, Cyperaceae, D. stramonium, Dianthus sp., E. citriodora, H. rosa-sinensis, H. integrifolia, $H$. auriculata, J. simplex, Lathyrus sp., L. esculentum, M. oleifera, M. alba, $O$. sanctum, P. hysterophorus, $P$. zeylanica, Poaceae, $P$. guajava, $R$. sativus, $S$. arvensis, Solanum sp., Tagetes sp., T. grandifolia, Vernonia sp. and Verbena sp. (Tab. 2).

Brassica campestris and $C$. sativum were very frequent, 16 pollen types were recorded as frequent, and the remaining 25 were infrequent in the total honey sample (Fig. 5). B. campestris was predominant in four samples

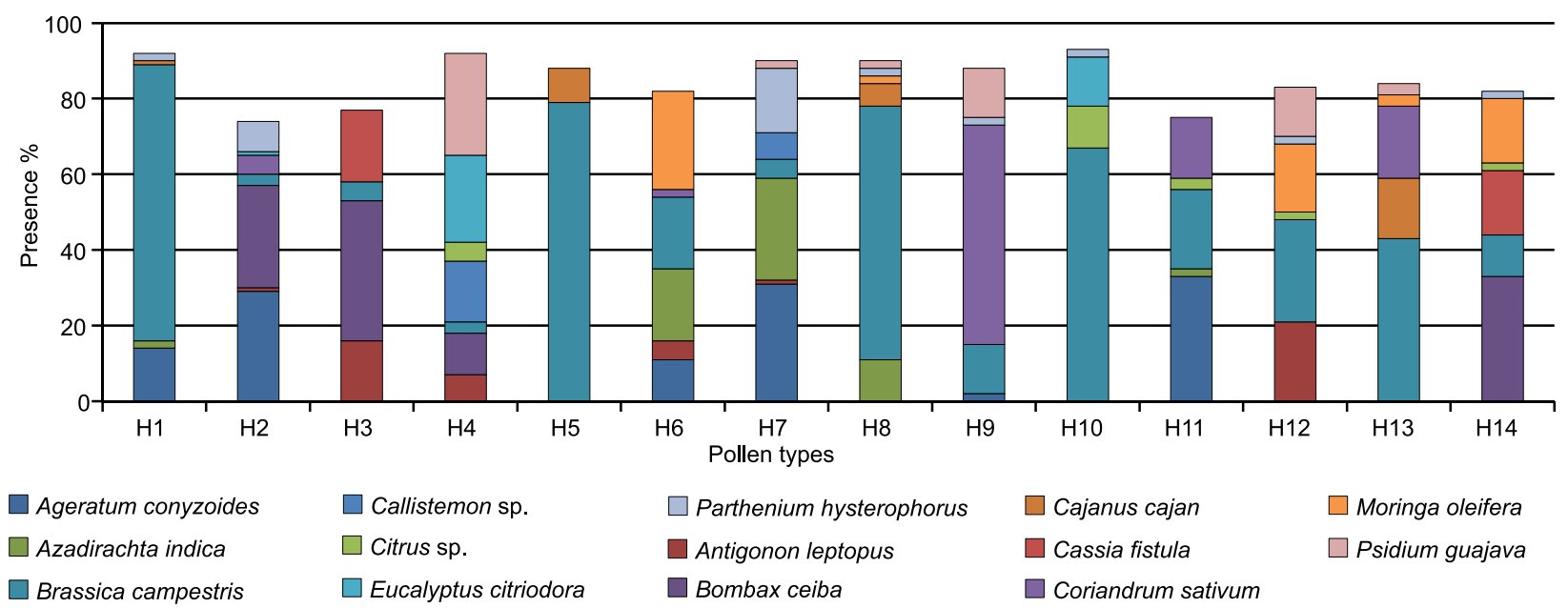

Fig. 4. Pollen types of main nectariferous plants of each honey sample 


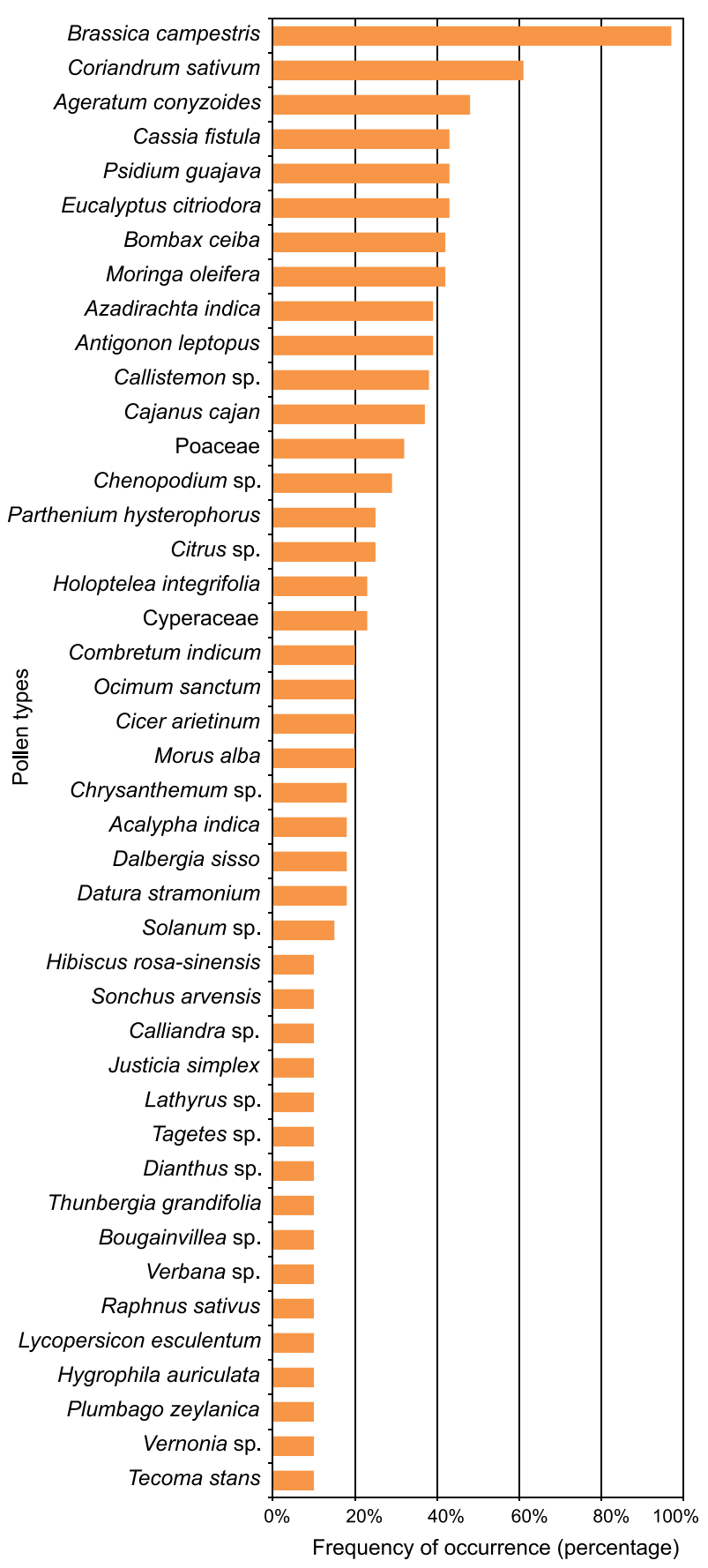

Fig. 5. Frequency of occurrence of pollen types recovered from the total honey samples collected from Prayagraj District, Uttar Pradesh

(H1, H5, H8, H10), secondary in four samples (H6, H9, H11, H12, H14) and an important minor pollen type in five samples $(\mathrm{H} 2, \mathrm{H} 3$, H4, H7, H13). B. campestris was documented as the main bee-forage plant. Similar observations have been published from different regions of India (Chauhan and Trivedi, 2011; Sahney and Rahi, 2015; Sahney et al., 2018). C. sativum was predominant in one sample (H9), secondary in two samples (H12, H13) and an important minor pollen type in one sample (H2).
Among the frequent types in Prayagraj District, A. conyzoides, A. indica, B. ceiba, Callistemon sp., Citrus sp. and E. citriodora are major nectariferous/ polleniferous plant taxa documented in different states of India (Datta et al., 2008; Pal and Karmarkar, 2013; Ramakrishna and Swathi, 2013; Chauhan et al., 2017; Sahney et al., 2018).

The pollen spectra of the 14 honey samples collected from different localities in Prayagraj District showed a diversity of pollen types, ranging in number from 7 to 16 (Fig. 2) and reaching maximum in multifloral honey sample H2 (16 pollen types), followed by H4 (15), H6 (14), H11 (14), H12 (14), H14 (14), H3 (13), H7 (13) and H13 (13). Minimum pollen diversity was recorded in unifloral sample H10 (7 pollen types), followed by $\mathrm{H} 1$ (8), H5 (8), H9 (8) and H8 (9).

With respect to absolute pollen count, four samples belonged to Gp II (poor: H1, H8, H10, H14), eight samples to Gp III (rich: H2, H3, H4, H5, H7, H9, H12, H13) and two samples to Gp IV (very rich: H6, H11).

The investigated region is mainly tropical, where the flowering of herbs, shrubs, climbers and trees is abundant during the winter season. These plants are profusely distributed along the extensive cultivated land in rural areas; in urban areas, tree avenues are common, as are herb and shrub plantings. In the present study we found that herbs (A. conyzoides, B. campestris, C. sativum, Chenopodium sp., J. simplex, P. hysterophorus), shrubs (Citrus sp., Callistemon sp., T. stans), climbers (A. leptopus, C. indicum, T. grandifolia) and trees (B. ceiba, E. citriodora, M. alba, P. guajava) were the main forage plants of Apis dorsata in Prayagraj District.

\section{PHYSICOCHEMICAL ANALYSIS}

Table 3 gives the results of physicochemical analyses for $\mathrm{pH}$, electrical conductivity, total dissolved solids, moisture content, ash content and mineral content. Results for honey color are given in Table 2. The values of these parameters are summarized below.

The $\mathrm{pH}$ of all honey samples ranged from $3.40 \pm 0.15$ to $4.74 \pm 0.4$ (Tab. 3). The low $\mathrm{pH}$ inhibits the growth of microorganism and influences the texture and stability of honey (Terrab et al., 2003). Low $\mathrm{pH}$ (3.49-4.70) of honey samples has also been reported from different regions of India, Argentina, Algeria and Estonia 


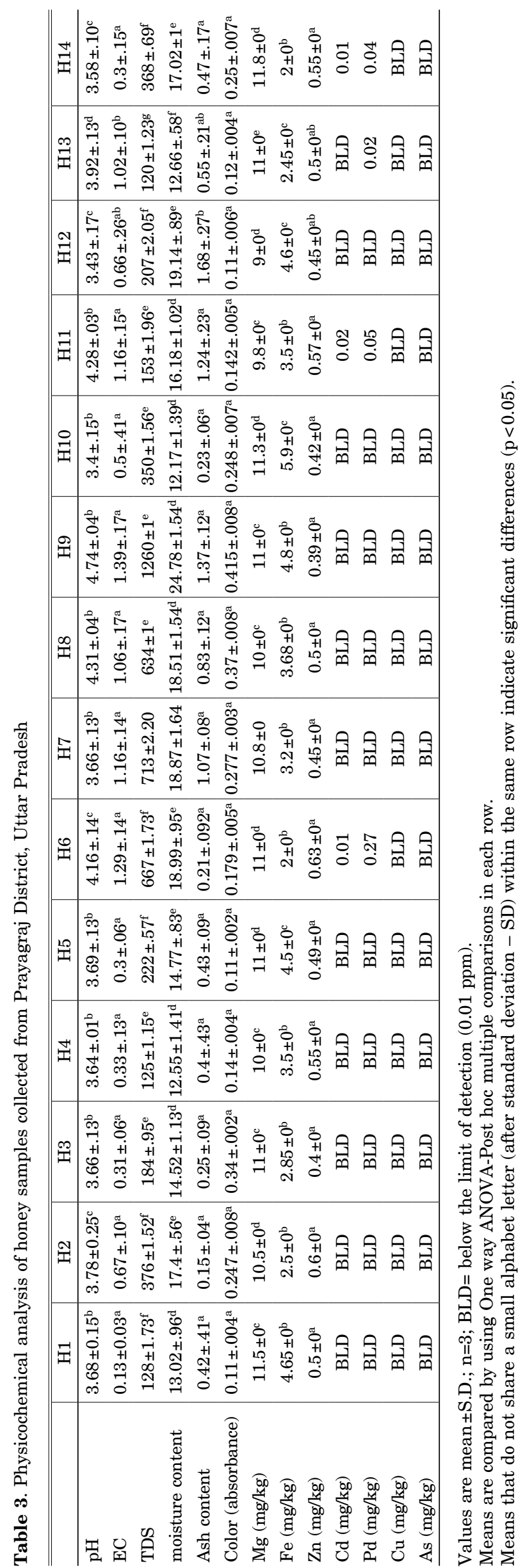

(Ouchemoukh et al., 2007; Cantarelli et al., 2008; Saxena et al., 2010; Kirs et al., 2011).

Electrical conductivity is a good criterion of the botanical origin of honey. Blossom honey and mixtures of blossom and honeydew honey should have conductivity of less than $0.8 \mathrm{mS} / \mathrm{cm}$ (Codex Alimentarius 2001). All the honey samples had electrical conductivity values ranging from $0.13 \pm 0.03 \mathrm{mS} / \mathrm{cm}$ to $1.39 \pm 0.17 \mathrm{mS} / \mathrm{cm}$.

Total dissolved solids (TDS) is a measure of the combined content of all inorganic and organic substances in honey in molecular, ionized or micro-granular (colloidal solution) suspended forms. All the honey samples had total dissolved solid values ranging from $120 \pm 1.23 \mathrm{ppm}$ to $1260 \pm 1 \mathrm{ppm}$.

The moisture content of all honey samples ranged from $12.17 \pm 1.39 \mathrm{mg} / 100 \mathrm{~g}$ to $19.14 \pm .89 \mathrm{mg} / 100 \mathrm{~g}$, except for sample H9, which measured $24.78 \pm 1.54 \mathrm{mg} / 100 \mathrm{~g}$ (Tab. 3 ). The recommended limit for moisture content of honey samples is $20 \mathrm{mg} / 100 \mathrm{~g}$ according to international quality regulations (Codex Alimentarius, 2001). Low moisture content prevents fermentation of honey and attack by microorganisms. It helps in its preservation and storage and increases the shelf life of honey (Buba et al., 2013; El-Metwally, 2015). Higher moisture content can lead to undesirable fermentation of honey during storage (Saxena et al., 2010). Moisture content depends on environmental factors during honey production, such as weather and hive humidity. It also depends on nectar conditions and on the treatment of honey during extraction and storage.

The ash content of the all honey samples varied between $0.15 \pm .04$ and $1.68 \pm .27 \mathrm{mg} / 100 \mathrm{~g}$, which is an acceptable range (Codex Alimentarius 2001). These results reflect the abundance of pollen sources in the vicinity of the bee hive sites during honey production. Similar observations have been made by various workers from different regions of India (Nanda et al., 2003; Sahney and Kumar, 2017), Turkey (Unal and Kuplulu, 2006), Argentina (Cantarelli et al., 2008) and Estonia (Kirs et al., 2011).

The color of the studied honey ranged from water-white to dark amber (Tab. 2). One honey sample from Prayagraj District was darker amber in color and had the highest Pfund value (H9). The other honey samples were water-white to amber in color: water-white in samples H1, H5, H12 and H13 (2.5129 mm Pfund), extra white in H14 and 
H11 (13.2946 and $14.0373 \mathrm{~mm}$ Pfund), white in sample H6 (27.77 mm Pfund), light amber in H14 (54.1475 mm Pfund), light amber in H7 (64.1750 mm Pfund), amber in H3 and H8 (87.5726 and $98.7143 \mathrm{~mm}$ Pfund) and dark amber in H9 (115.4268 mm Pfund). The color of honey depends on its ash content, temperature and storage time (De Silva et al., 2016).

Among the minerals, magnesium content was the highest, ranging from $9.0 \pm 0.0$ to $11.8 \pm 0 \mathrm{mg} / \mathrm{kg}$, followed by iron $(2.0 \pm 0.0$ to $5.9 \pm 0.0 \mathrm{mg} / \mathrm{kg})$ and zinc $(0.39 \pm 0.0$ to $0.6 \pm 0.0 \mathrm{mg} / \mathrm{kg}$ ). The heavy metals cadmium $(\mathrm{Cd})$, lead $(\mathrm{Pd})$, copper $(\mathrm{Cu})$ and arsenic $(\mathrm{As})$ were below the limit of detection $(<0.01 \mathrm{mg} / \mathrm{kg})$ in all samples except in $\mathrm{H} 6(\mathrm{Cd} 0.01 \mathrm{mg} / \mathrm{kg}, \mathrm{Pd}$ $0.27 \mathrm{mg} / \mathrm{kg}$ ), H11 (Cd $0.02 \mathrm{mg} / \mathrm{kg}, \mathrm{Pd} 0.05 \mathrm{mg} / \mathrm{kg}$ ), $\mathrm{H} 13(\mathrm{Pd} 0.02 \mathrm{mg} / \mathrm{kg})$ and $\mathrm{H} 14(\mathrm{Cd} 0.01 \mathrm{mg} / \mathrm{kg}$, $\mathrm{Pd} 0.04 \mathrm{mg} / \mathrm{kg})$.

\section{CONCLUSION}

In this study we conducted melissopalynological and physicochemical analyses of honey samples collected from rural and urban localities of Prayagraj District, Uttar Pradesh. We found unifloral honey mostly in rural areas, while multifloral honey was found in urban areas. Cultivated rural land is dominated by Brassica sp. crops, with sparse trees, while urban areas have tree avenues along with herb and shrub plantings. Our work provides information related to the pattern of vegetation of Prayagraj District. The studied honey can be considered as of good quality, as all samples were found to meet the guidelines of Codex Alimentarius (2001) and the European Commission (2002) for all physicochemical parameters ( $\mathrm{pH}, \mathrm{EC}, \mathrm{TDS}$, moisture content, ash content and color).

Our analyses indicate that Prayagraj District has a rich flora conducive to the production of good-quality honey. The study should be of use in promoting the commercialization of regional honey and in establishing the apiary industry in Prayagraj District, Uttar Pradesh.

\section{ACKNOWLEDGEMENTS}

Vibhasa Shukla is grateful to the University Grant Commission, New Delhi, India, for financial support under a female-candidate postdoctoral fellowship, and to Prof. K.S. Rao, Head of the Botany Department, University of Delhi, for providing the necessary facilities for this work. Ajay Kumar is grateful to the
National Academy of Sciences, Prayagraj, for providing financial support under a Senior Scientist Platinum Jubilee Research Associateship.

\section{REFERENCES}

AOAC, 1999. Official methods of analysis of AOAC international (P. Cunniff, ed.) $16^{\text {th }}$ ed. Revision. Vol. II. Association of Analytical Chemists. USA.

Bianchi, E.M., 1990. Quality control of honey and wax. UN for Agriculture and Food. FAO Agricultural Service Bulletin 68/3. Rome. p. 69.

Bogdanov, S., Martin, P., Lüllmann, C., 1997. Harmonised methods of the European honey commission. Apidologie, 1-59.

Buba, F., Gidado, A., Shugaba, A., 2013. Analysis of biochemical composition of honey samples from North-East Nigeria. Biochemistry and Analytical Biochemistry 2(3), 139.

Cantarelli, M.A., Pellerano, R.G., Marchevsky, E.J., Camiña, J.M., 2008. Quality of honey from Argentina: Study of chemical composition and trace elements. Journal of the Argentine Chemical Society 96(1-2), 33-41.

Chauhan, M.S., Trivedi, A., 2011. Pollen analysis of honey from Lucknow district, Uttar Pradesh. Journal of Applied Biosciences 37(1), 48-51.

Chauhan, M.S., Farooqui, A., Trivedi, A., 2017. Plant foraged by bees for honey production in northern India: The diverse flora of India and its implications for apiculture. Acta Palaeobotanica 57(1), 119-132.

Cherian, K.J., Bhowal, M., Godghate, S.D., 2011. Pollen and physicochemical analysis of honey produced by Apis cerana indica of nagpur, Maharashtra (India). Journal of Environmental Research And Development 5(3), 542-550.

Codex Alimentations, 2001. Draft revised standard for standard for honey (at step 10 of the Codex procedure). Alinorm. 01(25), 19-26.

Cotte, J.F., Casabianca, H., Chardon, S., Lheritier, J., Grenier-Loustalot, M.F., 2004. Chromatographic analysis of sugars applied to the characterisation of monofloral honey. Analytical and Bioanalytical Chemistry 380, 698-705.

Datta, K., Verma, P.C., Chatterjee, A., 2008. Mapping of key bee flora of upper Gangetic region of India - a palynological assessment through regional honeys. Grana 47, 159-170.

De Silva, P.M., Gauche, C., Gonzaga, L.V., Costa, A.C.O., 2016. Honey: Chemical composition, stability and authenticity. Food Chemistry 196, 309-323.

El-Metwally, A.A.E., 2015. Factors Affecting the Physical and Chemical Characteristics of Egyptian Beehoney. Ph. D. Thesis, Fac. Agric. Cairo Univ., 320 p.

European Commission. 2002. Council Directive $2001 / 110 / \mathrm{EC}$ of $20^{\text {th }}$, December 2001 relating to honey. Official Journal of the European Communities 10, 47-52. 
Feller-Demalsy, M.-J., Parent, J., Strachan, A.A., 1987. Microscopic analysis of honeys from Alberta. Canada. Journal of Apicultural Research 26(2), 123-132.

Feller-Demalsy, M.-J., Parent, J., Strachan, A.A, 1989. Microscopic analysis of honey from Manitoba, Canada. Journal of Apicultural Research 28, 41-49.

Gairola, A., Tiwari, P., Tiwari, J.K., 2013. Physicochemical properties of Apis cerana-indica f. honey from Uttarkashi district of Uttarakhand, India. Journal of Global Biosciences 2(1), 20-25.

Khatija, F., Ramanujan, C.G.K., 1993. Pollen and chemical characterization of Apis cerana and A. florae honeys from Hyderabad urban complex. Journal of Palynology 29, 59-67.

Kirs, E., Pall, R., Martverk, K., Laos, K., 2011. Physicochemical and melissopalynological characterization of Estonian summer honeys. Procedia Food Science 1, 616-624. doi: 10.1016/j.profoo.2011.09.093

Louveaux, J., Maurizio, A., Vorwohl, G., 1978. Methods of melissopalynology. Bee World 59, 139-157.

Nanda, V., Sarkara, B.C., Sharma, H.K., Bawa, A.S.V., 2003. Physicochemical properties and estimation of mineral content in honey produced from different plants in Northern India. Journal of Food Composition and Analysis 16, 613-619.

Ouchemoukh, S., Louaileche, H., Schweitzer, P., 2007. Physicochemical characteristics and pollen spectrum of some Algerian honeys. Food Control 18, 52-58.

Pal, P.K., Karmarkar, K., 2013. Pollen analysis in understanding the foraging behaviour of Apis mellifera in Gangetic West Bengal. Geophytology 42, 93-114.

Pereira, P.C.M., Barraviera, B., Burini, R.C., Soares A.M.V.C., Bertani, M.A., 1995. Use of honey as nutritional and therapeutic supplement in the treatment of infectious diseases. Journal of Venomous Animals and Toxins. Preliminary Report 1, 1-2.

Ponnuchamy, R., Bonhomme, V., Prasad, S., Das, L., Patel, P., 2014. Honey pollen: using melissopalynology to understand foraging preferences of bees in tropical South India. PLoS ONE 9(7), 1-11.
Ramakrishna, H., Swathi, S., 2013. Pollen diversity in some Apis florae honeys from Adilabad District. Andhra Pradesh, India. Geophytology 42, 11-20.

Ramnath, S., Venkataramegowda, S., 2012. Physicochemical and pollen analysis of Western Ghats honey of Karnataka, South India. International Journal of Sciences and Nature 3(4), 831-835.

Sahney, M., Kumar, A., 2017. Physiochemical and mineral analysis of honey samples from Varanasi district. International Journal of Pharma and Bio Sciences 8(2), 160-166

Sahney, M., Rahi, S., 2015. Pollen analysis of honey samples from Allahabad district, Uttar Pradesh, India. Geophytology 45(1), 21-30.

Sahney, M., Rahi, S., Kumar, A., Jaiswal, R., 2018. Melissopalynological studies on winter honeys from Allahabad, Uttar Pradesh. Palynology 42(4), 540-552.

Saxena, S., Gautam, S., Sharma, A., 2010. Physical, biochemical and antioxidant properties of some Indian honeys. Food Chemistry 118, 391-397.

Shobham, Chitluri, K.K., Nayar, J., 2017. PhysicoChemical Analysis of Some Commercial Honey Samples from Telangana. Indian Journal of Nutrition 4(1), 150-153.

Suryanarayana, M.C., Seethalakshmi, T.S., Phadke, R.P., 1981. Pollen analysis of Indian honeys 1. Honeys from Litchi (Nephelium litchi) and Jamun (Syzygium cumini). Proc. IV Int. Palynol. Conf. Luc. (1976-77) 3, 491-498.

Terrab, A., Diez, M.J., Heredia, F.J., 2003. Palynological, physicochemical and color characterization of Moroccan honeys: I. River red gum (Eucalyptus camaldulensis Dehnh) honey. International Journal of Food Science \& Technology 38, 379-386.

Unal, C., Kuplulu, O., 2006. Chemical quality of strained honey consumed in Ankara. Ankara Universitesi Veteriner Fakultesi Dergisi 53, 1-4.

Wodehouse, R.P., 1935. Pollen grains, their structure, identification and significance in science and medicine. New York and London: McGraw Hill Book Company, Inc. 